\title{
In-vitro proteinase production by oral Candida albicans isolates from individuals with and without HIV infection and its attenuation by antimycotic agents
}

\author{
T. WU, L. P. SAMARANAYAKE ${ }^{\dagger}$, B. Y. CAO* and J. WANG* \\ Oral Biology Unit, Faculty of Dentistry, University of Hong Kong, 34 Hospital Road, Hong Kong and \\ *Department of Biochemistry, Chinese University of Hong Kong, New Territories, Hong Kong
}

\begin{abstract}
In-vitro proteinase production by oral Candida albicans isolates from patients with and without HIV infection (18 isolates from each group) was assessed by image analysis of a plate assay, with bovine serum albumin (BSA) as a substrate. The effect of sub-minimal inhibitory concentrations (sub-MICs) of nystatin, amphotericin B, clotrimazole and miconazole on in-vitro proteinase production by these yeast isolates was also investigated. Proteinase production by $C$. albicans isolates from patients with HIV infection was significantly greater than production by those from individuals without infection. All 18 isolates from HIV-infected individuals produced proteinase, in comparison to $56 \%$ of isolates from uninfected individuals. Pre-exposure of $C$. albicans isolates (seven proteinase producers from each group) to 1/4 and 1/16 MICs of nystatin, amphotericin B, clotrimazole and miconazole resulted in decreased proteinase production in all isolates tested. However, after exposure to the four antimycotic agents, proteinase production was decreased to a significantly greater extent in isolates from uninfected individuals than in those with HIV disease. Furthermore, when the relative concentration effect of antimycotic agents on proteinase production was compared, $C$. albicans isolates from the HIV-free group demonstrated a salient dose-response relationship compared with the HIV-infected group. These results indicate that $C$. albicans from patients with HIV infection are significantly more proteolytic than those from individuals without the infection, and that polyenes and imidazoles curtail the proteolytic activity of all $C$. albicans isolates, albeit to a lesser extent in those from HIVinfected patients. It appears that HIV disease favours oral colonisation by more proteolytic $C$. albicans isolates, with resilient proteolytic activity.
\end{abstract}

\section{Introduction}

Extracellular proteinase is considered to be a virulence factor of $C$. albicans [1-3], the most common agent of human candidosis. For example, immunoglobulin A, one of the main mucosal defence barriers, can be rapidly and thoroughly cleaved by proteinase [4]. It has also been reported recently that complement $\mathrm{C}_{3}$ and some specific endogenous proteinase inhibitors involved in regulating inflammation can also be degraded by $C$. albicans proteinase [5]. Proteinase of $C$. albicans can digest, in vitro, several physiologically important substances including albumin, keratin and skin proteins $[3,6,7]$. The in-vivo secretion of proteinase has been demonstrated in infected tissues by histochemical

Received 2 June 1995; revised version accepted 23 Aug. 1995.

${ }^{\dagger}$ Correspondence should be sent to Dr L. P. Samaranayake. methods [8,9]; moreover, secretion of proteinase correlates strongly with other virulence traits of C. albicans, such as adherence to epithelia [10]. The foregoing suggest the importance of Candida proteinase in the pathogenesis of candidosis.

The extracellular proteinase of $C$. albicans, first described by Staib [11], is not a single entity but is known to consist of three distinct enzymes [4], a complete proteinase and two that are partially proteolytic. Although the latter enzymes differ from the complete proteinase in substrate specificity and other properties, they cross-react immunologically with each other. These enzymes are classified as aspartate proteinases (EC 3.4.23.6) and are related to pepsin, renin and cathepsin $\mathrm{D}$. Their mol.wt is $c .45 \mathrm{kDa}$, with optimal activity in the $\mathrm{pH}$ range $3.5-4.0$ [12].

It is recognised that oral candidosis caused by 
C. albicans is one of the most common infections associated with human immunodeficiency virus (HIV) infection [13, 14]. Nevertheless, little is known of the production profile of the extracellular proteinase of $C$. albicans from HIV-infected patients in comparison with those from disease-free individuals. Very recently it has been observed that some isolates of $C$. albicans from HIV-infected patients are more proteolytic than those from HIV-uninfected individuals [15, 16].

Furthermore, the effect of major antimycotic agents polyenes and imidazoles - on in-vitro proteinase production by $C$. albicans has not been studied systematically. Angiolella et al. [17] investigated this phenomenon and noted that methyl partricin, fenticonazole and 5-fluorocytosine suppressed proteinase production by $C$. albicans, but no other reports of this phenomenon are documented.

Accordingly, two related investigations were performed in the current study, with two groups of 18 oral isolates of $C$. albicans, from patients with and without HIV infection. Firstly, in-vitro proteinase production by oral C. albicans isolates from these two groups was compared, and secondly, the effect of antimycotic agents on proteinase secretion of a randomly selected group of 14 isolates was assayed by exposing them to $1 / 4$ and $1 /$ 16 sub-MICs of two polyenes (nystatin and amphotericin B) and two imadazoles (clotrimazole and miconazole).

\section{Materials and methods}

\section{Candida isolates and growth conditions}

Two gromps comprising 18 oral $C$. albicans isolates each were used in the study. The isolates in the first group were from patients with HIV infection whose CD4 (cell counts were $<400$ cells $/ \mathrm{mm}^{3}$, attending the $\mathbb{H E I V}$ Research Clinic of the Faculty of Dentistry, University of Hong Kong, Hong Kong. The second group of 118 isolates was from HIV-free patients anith angular cheilitis, obtained by the Microbiology Researcth Services of the same institution. These isolates were identified by sugar assimilation and fermentation tests with the API 20C system (API products, bioMérieux, Lyon, France) and the 'germ tube' test. Stock cultures were maintained at $4^{\circ} \mathrm{C}$ on Sabouraud dextrose agar (SDA). All isolates were first screened for proteinase production by the bovine serum albumin (BSA) assay; proteinase-positive isolates (seven from each group) were subsequently selected at random for tests with antimycotic agents.

\section{Quantification of proteinase production by the BSA assay}

Extracellular proteinase production by $C$. albicans was induced with BSA according to the technique described by Staib [11]. Briefly, an 18-h yeast suspension of $1 \times 10^{6} \mathrm{cells} / \mathrm{ml}$ was prepared, and $5 \mu \mathrm{l}$ of this yeast suspension were spot inoculated on to a BSA $1 \%$ agar plate. The plate was incubated aerobically in a humidified container for 5 days at $37^{\circ} \mathrm{C}$, flooded with naphthalene black $1.25 \%$ solution in methanol/water $90 \% \mathrm{v} / \mathrm{v}$ for $15 \mathrm{~min}$ and decolourised for a further $36 \mathrm{~h}$ with several changes of the latter solution. The diameter of the zone proteolysis and the colony were examined and quantified by Quantimet 500+ Image Analysis System (Quantimet Image System, Leica Instruments, Hong Kong). Proteinase production $\left(\operatorname{Pr}_{d}\right.$ value) was calculated as follows:

$$
\operatorname{Pr}_{\mathrm{d}} \text { value }=\mathrm{D}_{\mathrm{z}} / \mathrm{D}_{\mathrm{c}}
$$

(where $\mathrm{D}_{z}$ was the diameter of the zone of proteolysis and the colony, $\mathrm{D}_{c}$ was the diameter of the colony).

All the experiments were repeated on three separate occasions with duplicate determinations on each occasion.

\section{Preparation of antimycotic agents}

Antimycotic agents used were as follows: nystatin, amphotericin B, clotrimazole and miconazole (Sigma). Nystatin and amphotericin B were dissolved in a mixture of dimethyl sulphoxide and ethanol (3:2); clotrimazole and miconazole were dissolved in $\mathrm{N}$ dimethylformamide. MICs of antimycotic agents used were $(\mu \mathrm{g} / \mathrm{ml})$ : nystatin 2.0 , amphotericin B 0.4 , colotrimazole 110:0, miconazole 4.0 [18]. Dilutions of $1 / 4$ and $1 / 16$ of the above concentrations were used in the experiments.

\section{Ewaluating the effect of antimycotic agents on pmoteinase production by $C$. allbicans}

To thest the effect of antimycotic agents on proteinase production by $C$. alficons, a loopful of 18-h yeast growth was transferred to $10 \mathrm{ml}$ of yeast nitrogen base medium supplemented with $50 \mathrm{mM}$ glucose with or withou ubinhibitory concentrations of $1 / 4$ and $1 / 16$ MIC dilutions of antimycotics, and grown aerobically at $37^{\circ} \mathrm{C}$ in a shaking water bath for $18 \mathrm{~h}$. The cells were harvested by centrifuging at $1500 \mathrm{~g}$ for $10 \mathrm{~min}$, washed twice in $10 \mathrm{ml}$ of sterile phosphate-buffered saline $(0.1 \mathrm{M}, \mathrm{pH} 7.2)$ and resuspended in the same buffer to a final concentration of $1.0 \times 10^{6}$ yeasts $/ \mathrm{ml}$ as determined by microscopic counting in a haemocytometer counting chamber. The subsequent procedure for inducing proteinase production on BSA was as described above.

\section{Statistical analysis}

All the numerical data obtained were analysed by the Wilcoxon matched-pairs signed-ranks test. 


\section{Results}

Assessment of proteinase activity by the image analysis system

In pilot studies both visual and computerised image analysis measurement were used to record the diameter of the zone of proteolysis and the fungal colony. As highly concordant and accurate results were obtained once the image analysis system was standardised, the latter was used subsequently throughout the study. The replicate $\mathbf{P x}_{d}$ data for each isolate were highly reproducible, as shown by the very low standard deviation (range $0.0-8.4 \%$ ); the highest coefficient of variation was $<9 \%$.

\section{In-vitro proteinase production by $C$. albicans} isolates from patients with and without HIV infection

C. dlbicans isolates from HIV-infected patients were significantly more proteolytic than those from HIVuninfected individuals $\left(\mathrm{p}=0.002\right.$, Fig. 1). The mean $\operatorname{Pr}_{d}$ value for the HIV group (1.73) was $25 \%$ higher than that for the HIV-free group (1.29). It was also noticeable that all 18 isolates in the former group were proteinase positive, in contrast to $56 \%$ isolates in the latter group.

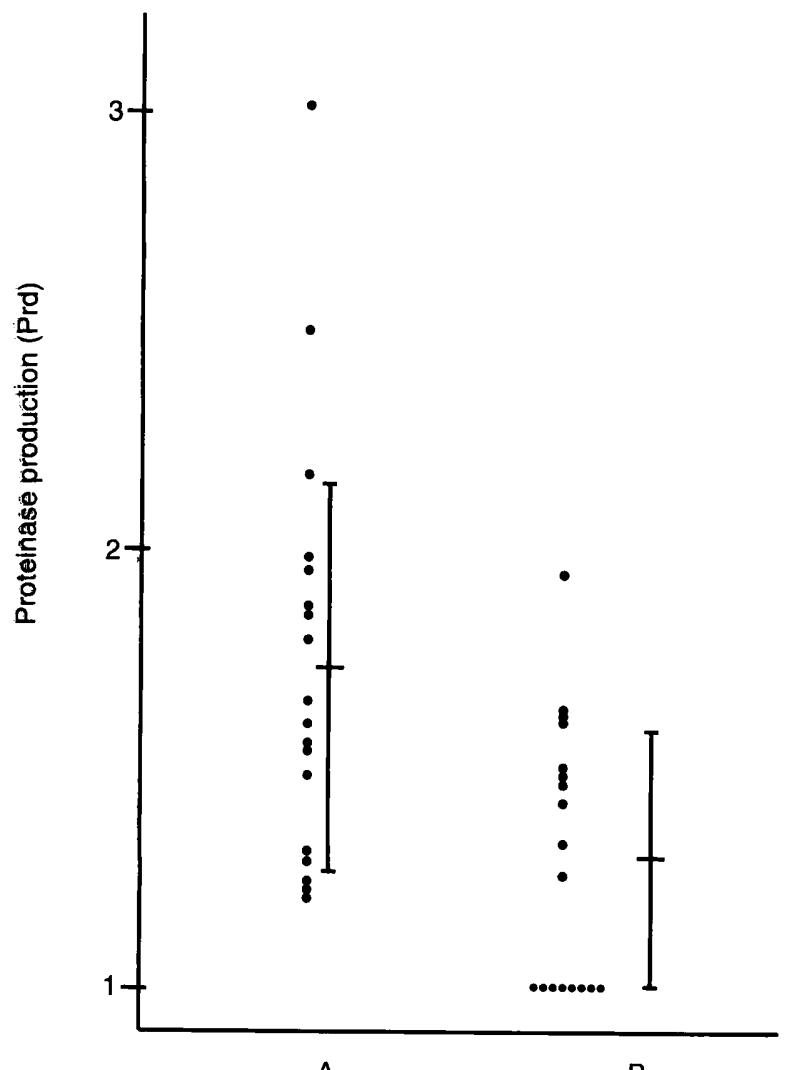

A

B

Fig. 1. Proteinase production by $C$. albicans isolated from (A) HIV-infected (mean 1.73) and (B) -uninfected (mean 1.29) individuals $(p=0.002)$. Proteinase production $\left(\operatorname{Pr}_{d}\right)$ was expressed as a function of diameter of the zone of proteolysis and the colony (see text).
Effect of antimycotic agents on in-vitro proteinase production

The effect of four different antimycotic agents on proteinase production of $C$. albicans from individuals with or without HIV infection is shown in Fig. 2. Compared with the control groups (no antimycotic treatment) exposure to sub MICs of nystatin, amphotericin B, clotrimazole and miconazole significantly decreased proteinase production by all $14 \mathrm{C}$. albicans isolates tested ( $p=0.008$; Fig. 2 , right-hand column). Of the four antimycotic agents tested, exposure to subMICs of miconazole resulted in the greatest reduction in proteinase production $(22.35 \%)$, followed by nystatin (21.18\%), amphotericin B (16.47\%) and clotrimazole $(15.88 \%)$.

When the effect of antimycotic agents on isolates from the HIV-infected and non-infected groups was compared, it was clear that proteinase production was reduced to a significantly greater extent in the isolates from the latter group (except exposure to 1/16 MIC of nystatin) (Table 1).

Generally, there was a good dose-response relationship between enzyme production and exposure to all four antimycotic agents in isolates from the disease-free group, i.e., exposure to $1 / 4 \mathrm{MIC}$ suppressed proteinase production to a greater extent than exposure to $1 / 16$ MIC ( $p<0.01$, Fig. 3). However, this phenomenon was less apparent in the isolates from the HIV group exposed to nystatin and clotrimazole. There was no significant difference in proteinase production between $1 / 4$ and $1 / 16$ MICs exposure of isolates to the two drugs ( $p>0.05$, Fig. 3).

\section{Discussion}

Candida infections are found in at least $80 \%$ of patients with acquired immunodeficiency syndrome (AIDS) and in about one third of those with HIV infection $[13,14,19]$. Oral candidosis is one of the most common fungal infections, appearing mainly in the oral cavity as erythematous or pseudomembranous lesions [13, 14]. C. albicans is undoubtedly the most common causative agent of this disease; its extracellular acid proteinase is a recognised virulence attribute, which facilitates tissue penetration, while proteinase-deficient strains are less invasive [3]. Whereas some have cast doubt on the relevance of candida proteinases in oral candidosis [20], others have demonstrated convincingly that conditions may prevail intra-orally that facilitate its activity $[21,22]$. Indeed, the acidic proteinase of $C$. albicans, which requires low $\mathrm{pH}$ for optimal activity, is active in acidic salivas that persist in certain physiological and pathological states (e.g., xerostomia, Sjögrens syndrome) [23]. Interestingly, xerostomia and accompanying changes in salivary composition and lower $\mathrm{pH}$ 


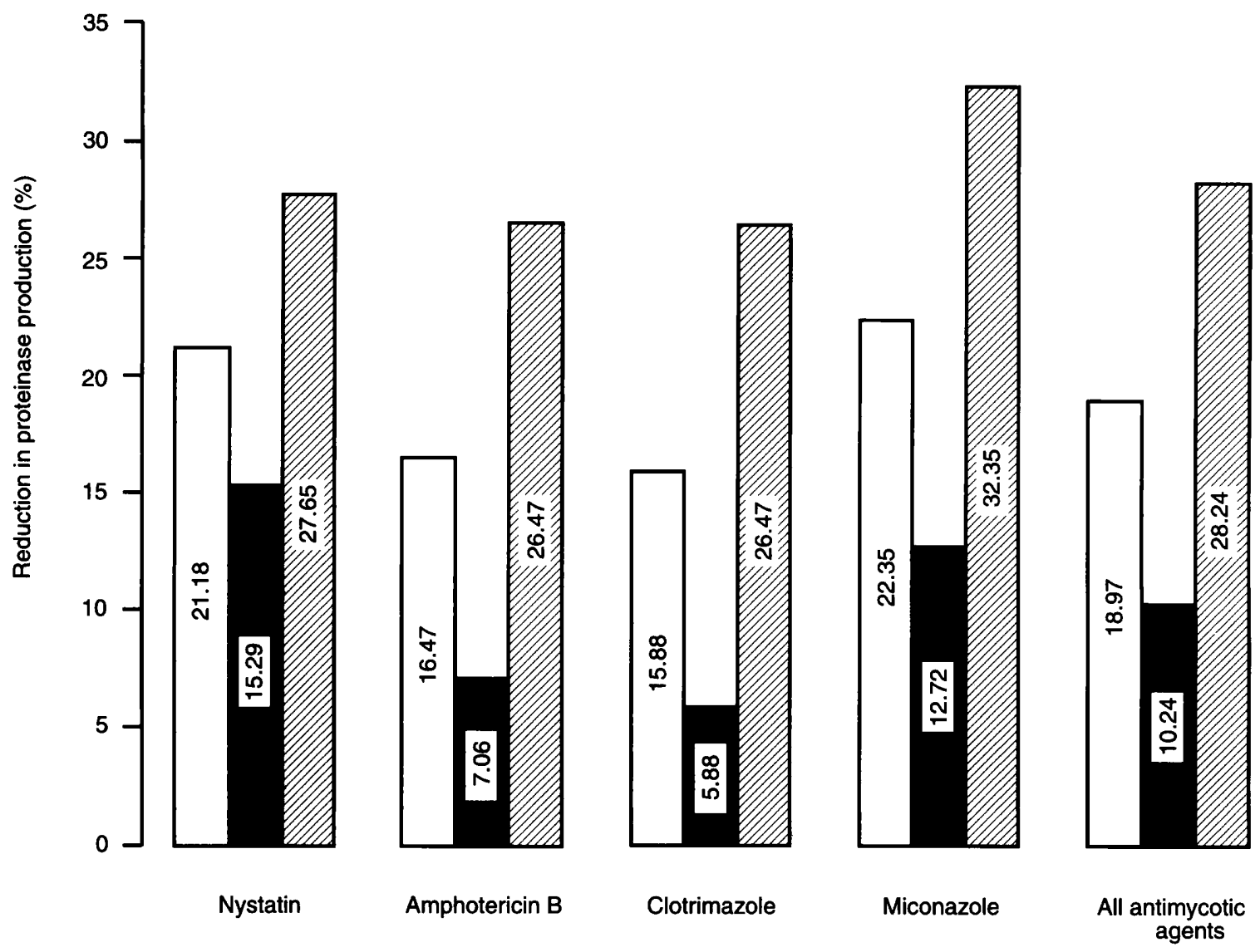

Fig. 2. Percentage reduction in proteinase production by $C$. albicans isolates from HIV-infected ( $\boldsymbol{\square})$ and -uninfected $(\square)$ individuals, and the total study population $(\square)$ after exposure to four different antimycotic agents.

Table 1. Differences in proteinase production between $C$. albicans isolates from HIV-infected and non-infected individuals, after exposure to sub-MICs of four antimycotic agents

\begin{tabular}{|c|c|c|c|c|c|c|c|c|}
\hline \multirow[b]{3}{*}{ Patient status } & \multicolumn{8}{|c|}{ Mean $\operatorname{Pr}_{\mathrm{d}}{ }^{*}$ by $C$. albicans strains exposed to } \\
\hline & \multicolumn{2}{|c|}{ Nystatin } & \multicolumn{2}{|c|}{ Amphotericin B } & \multicolumn{2}{|c|}{ Clotrimazole } & \multicolumn{2}{|c|}{ Miconazole } \\
\hline & $1 / 4^{+}$ & $1 / 16$ & $1 / 4$ & $1 / 16$ & $1 / 4$ & $1 / 16$ & $1 / 4$ & $1 / 16$ \\
\hline $\mathrm{HIV}^{+}$ & 1.42 & 1.46 & 1.47 & 1.68 & 1.58 & 1.62 & 1.40 & 1.56 \\
\hline HIV $^{-}$ & 1.16 & 1.29 & 1.13 & 1.36 & 1.14 & 1.36 & 1.05 & 1.25 \\
\hline $\mathrm{p}$ value & $<0.05$ & NS & $<0.05$ & $<0.01$ & $<0.05$ & $<0.05$ & $<0.05$ & $<0.01$ \\
\hline
\end{tabular}

NS, no significant difference.

${ }^{*}$ Seven isolates, each tested in duplicate on three occasions.

${ }^{\dagger}$ Sub-MIC concentration.

values have been recorded recently in HIV-infected patients [24-27].

Several methods, including BSA plate assay, spectrophotometry, SDS-PAGE and ELISA, can be used to evaluate $C$. albicans extracellular proteinase activity. Although proteinase activity assay on BSA plates has, up to now, been considered as a semi-quantitative method with visual estimation of the area of proteolysis [28], this technique has been complemented with an image analysis system to generate accurate and reproducible data, as shown in this investigation, where the highest coefficient of variation in replicate experiments was $<9 \%$.
The present results indicate that $C$. albicans isolates from HIV-infected individuals are more proteolytic than those from uninfected individuals. There are two recent reports on this phenomenon. Bernardis et al. [15], studied C. albicans isolates from 155 HIVinfected subjects by scoring the proteinase activity on BSA agar and by monitoring the secreted proteinase antigen by an immunoenzymatic assay. They found that $C$. albicans isolates from patients with advanced HIV infection were more proteolytic than those from either earlier stages of HIV infection or HIVuninfected subjects [15]. Others have found that genetically atypical $C$. albicans strains existed in HIV-infected individuals that could produce greater 


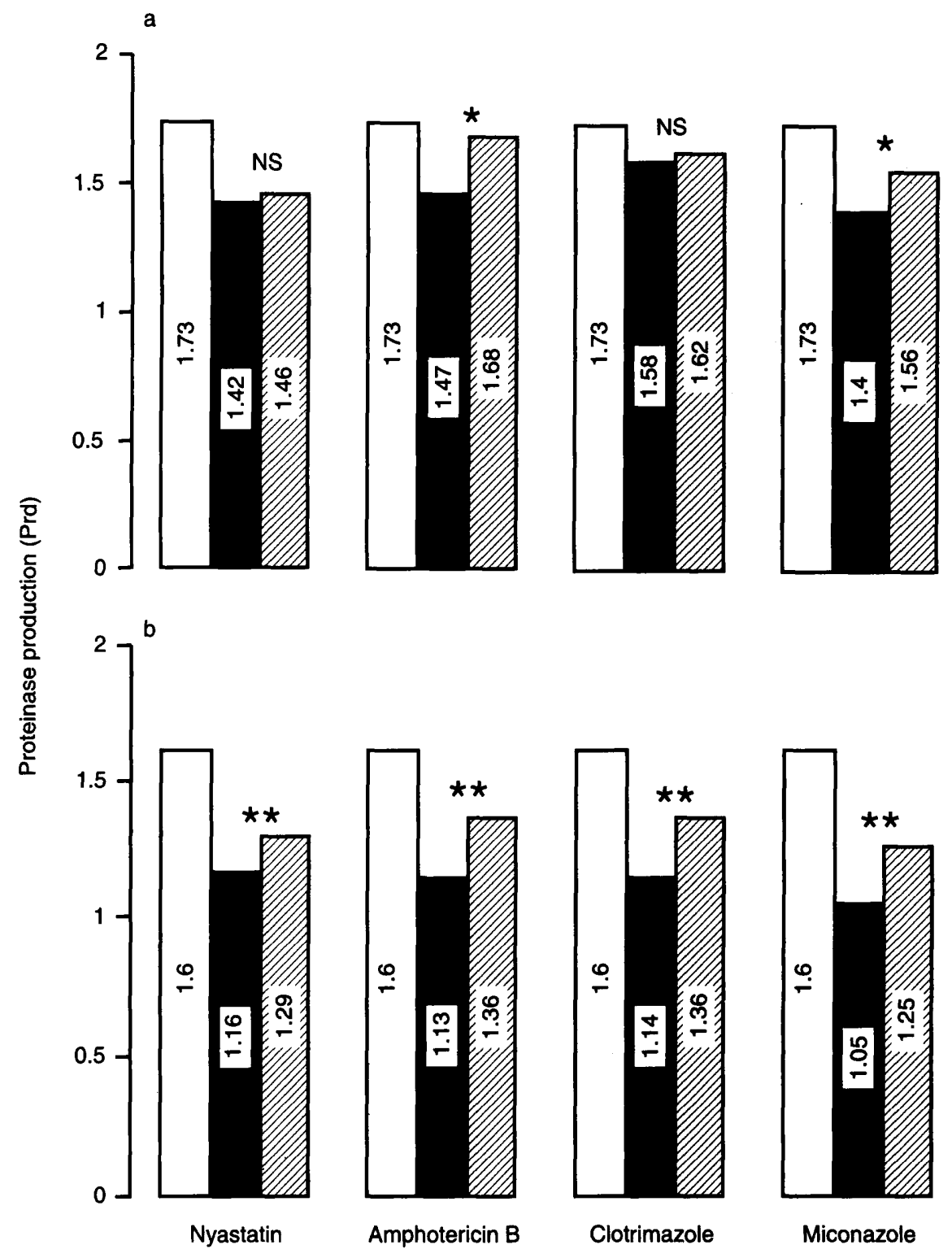

Fig. 3. Effect of pre-exposure to sub-MICs of four different antimycotic agents on proteinase production by $C$. albicans from (a) HIV-infected and (b) -uninfected individuals. Significant differences in proteinase production between isolates exposed to $1 / 4(\square)$ and $1 / 16(\square)$ MICs is denoted by asterisks, ${ }^{*} p<0.05 ;{ }^{* *} p<0.01$; NS, not significant; $\square$, controls without the antimycotic agent.

amounts of extracellular proteinase than typical C. albicans strains [16].

The results of the effect of polyenes (nystatin and amphotericin B) and imidazoles (clotrimazole and miconazole) on extracellular proteinase of $C$. albicans from individuals with and without HIV infection indicate that sub-MICs of these antifungal agents may modulate the proteolytic activity of $C$. albicans. This conclusion differs from that of the report by Angiolella et al. [17]. They found that only the polyene derivative methyl partricin suppressed proteinase production, but not the azoles miconazole, fluconazole and ketoconazole. This disparity is likely to be due to the fact that only a single isolate was used by the latter workers; 14 isolates were used in the current study.
The present results indicate that exposure to polyenes and imidazoles tends to have less inhibitory effect on proteinase production by $C$. albicans isolates from patients with HIV infection, when compared with isolates from patients without HIV infection. This suggests that proteinase production in C. albicans isolates in HIV disease is not only stronger but also more recalcitrant than in isolates from uninfected groups. Indeed, genetically atypical $C$. albicans isolates less susceptible to antimycotic agents have been described in HIV-infected patients [17], indicating that response to antifungal agents may be modulated in association with HIV disease. In clinical terms, this sequence of events might then be one reason for the well known treatment failure of oral and systemic antimycotic therapy of oral candidosis in HIV-infected and AIDS patients [14,29]. 
This research was supported by the University Grants Council of Hong Kong grant no. 338/268/0001.

\section{References}

1. Macdonald F, Odds FC. Virulence for mice of a proteinasesecreting strain of Candida albicans and a proteinase-deficient mutant. J Gen Microbiol 1983; 29: 431-438.

2. Rüchel R. Properties of a purified proteinase from the yeast Candida albicans. Biochem Biophys Acta 1981; 659: 99-113.

3. Kwong-Chung KJ, Lehman D, Good C, Magee PT. Genetic evidence for role of extracellular proteinase in virulence of Candida albicans. Infect Immun 1985; 49: 571-575.

4. Rüchel $R$, Tegeler $R$, Trost $M$. A comparison of secretory proteinases from different strains of Candida albicans. Sabouraudia 1982; 20: 233-244.

5. Kaminishi $\mathrm{H}$, Miyaguchi $\mathrm{H}$, Tamaki $\mathrm{T}$ et al. Degradation of humoral host defense by Candida albicans proteinase. Infect Immun 1995; 63: 984-988.

6. Negi $M$, Tsuboi $R$, Matsui $T$, Ogawa $H$. Isolation and characterization of proteinase from Candida albicans: substrate specificity. $J$ Invest Dermatol 1984; 83: 32-36.

7. Hattori $M$, Yoshiura $K$, Negi M, Ogawa H. Keratinolytic proteinase produced by Candida albicans. Sabouraudia 1984; 22: 175-183.

8. Macdonald F, Odds FC. Inducible proteinase of Candida albicans in diagnostic serology and in the pathogenesis of systemic candidosis. $J$ Med Microbiol 1980; 13: 423-435.

9. Rüchel R. A variety of Candida proteinases and their possible targets of proteolytic attack in the host. Zentralbl Backteriol Mikrobiol Hyg [A] 1984; 257: 266-274.

10. Datta A, Ganesan K, Natarajan K. Current trends in Candida albicans research. Adv Microbiol Physiol 1989; 30: 53-88.

11. Staib F. Serum-proteins as nitrogen source for yeastlike fungi. Sabouraudia 1965; 4: 187-193.

12. Rüchel R. Virulence factors of Candida species. In: Samaranayake LP, MacFarlane TW (eds) Oral candidosis. London, Wright-Butterworth. 1990: 47-65.

13. Samaranayake LP. Oral mycoses in HIV infection. Oral Surg Oral Med Oral Pathol 1992; 73: 171-180.

14. Holmstrup P, Samaranayake LP. Acute and AIDS-related oral candidoses. In: Samaranayake LP, MacFarlane TW (eds) Oral candidosis. London, Wright-Butterworth. 1990: 133-155.

15. De Bernardis F, Boccanera M, Rainaldi L, Guerra CE, Quinti
$\mathrm{L}$, Cassone A. The secretion of aspartyl proteinase, a virulence enzyme, by isolates of Candida albicans from the oral cavity of HIV-infected subjects. Eur J Epidemiol 1992; 8: 362-367.

16. McCullough M, Ross B, Reade PC. Oral Candida albicans from patients infected with the human immunodeficiency virus and characterization of a genetically distinct subgroup of Candida albicans. Austr Dent $J$ 1995; 40: 91-97.

17. Angiolella L, De Bernardis F, Bromuro C, Modello F, Ceddia T, Cassone A. The effect of antimycotics on secretory acid proteinase of Candida albicans. $J$ Chemother 1990; 2: 55-61.

18. McGinnis MG, Rinaldi MG. Antifungal drugs: mechanisms of action, drug resistance, susceptibility testing, and assays of activity in biological fluids. In: Lorian V (ed) Antibiotics in laboratory medicine, 3rd edn. Baltimore, Williams and Wilkins. 1991: 198-278.

19. Challacombe SJ. Immunologic aspects of oral candidiasis. Oral Surg Oral Med Oral Pathol 1994; 78: 202-210.

20. Germaine GR, Tellefson LM, Johnson GL. Proteolytic activity of Candida albicans: action on human salivary proteins. Infect Immun 1978; 22: 861-866.

21. Samaranayake LP, Hughes A, MacFarlane TW. The proteolytic potential of Candida albicans in human saliva supplemented with glucose. J Med Microbiol 1984; 17: 13-22.

22. Samaranayake YH, MacFarlane TW, Samaranayake LP, Aitchison $\mathrm{T}$. The in vitro proteolytic and saccharolytic activity of Candida species in human saliva. Oral Microbiol Immunol 1994; 9: 229-235.

23. Mason DK, Chisholm DM. Salivary glands in health and disease. London: W B Saunders. 1975: 410.

24. Atkinson JC, Yeh CK, Bermudez D, Fox PC, Baum BJ. Longitudinal evaluation of major salivary gland function in HIV-1 infected patients. $J$ Oral Pathol Med 1989; 18: 469-470.

25. Atkinson JC, Yeh CK, Oppenheim FG, Bermudez D, Baum BJ, Fox PC. Evaluation of salivary antimicrobial proteins following HIV-1 infection. Acquir Immune Defic Syndr 1990; 3: 41-48.

26. Mandel ID, Barr CE, Turgeon L. Longitudinal study of parotid saliva in HIV-1 infection. $J$ Oral Pathol Med 1992; 21: 209-213.

27. Marder MZ, Barr CE, Mandel ID. Cytomegalovirus presence and salivary composition in acquired immunodeficiency syndrome. Oral Surg Oral Med Oral Pathol 1985; 60: 372-376.

28. Ogrydziak DM. Yeast extracellular proteases. Crit Revi Biotechnol 1993; 13: 1-55.

29. Samaranayake LP. Superficial fungal infections. In: Scully C (ed) Current opinions in Dentistry. Philadelphia, Current Science. 1991: 415-422. 